Revue des sciences de l'éducation

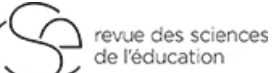

\title{
Le programme d'Histoire et éducation à la citoyenneté et \\ l'apprentissage de la pensée historique : une analyse \\ lexicométrique
}

The History and Citizenship Education Program and learning

historical thinking: a lexicometrical analysis

El programa de Historia y educación a la ciudadanía y el

aprendizaje del pensamiento histórico: un análisis

lexicométrico

Daniel Moreau

Volume 43, numéro 1, 2017

Texte reçu le : 7 avril 2016

Version finale reçue le : 22 février 2017

Accepté le : 16 mars 2017

URI : https://id.erudit.org/iderudit/1042077ar

DOI : https://doi.org/10.7202/1042077ar

Aller au sommaire du numéro

Éditeur(s)

Revue des sciences de l'éducation

ISSN

1705-0065 (numérique)

Découvrir la revue

Citer cet article

Moreau, D. (2017). Le programme d'Histoire et éducation à la citoyenneté et

l'apprentissage de la pensée historique : une analyse lexicométrique. Revue des sciences de l'éducation, 43(1), 141-178. https://doi.org/10.7202/1042077ar
Résumé de l'article

Cet article poursuit dans le sillage d'une analyse conceptuelle réalisée antérieurement. Dans cette analyse, nous interrogions le rapport des programmes d'histoire publiés au Québec depuis la réforme curriculaire entamée au début des années 1980 à l'apprentissage de la pensée historique. Cet article a pour objectif d'éclaircir ces divergences par une analyse lexicométrique du programme d'Histoire et éducation à la citoyenneté. Pour ce faire, une analyse factorielle des correspondances a été réalisée de manière à mettre au jour des traits structuraux spécifiques. Ces derniers rejoignent certaines propriétés associées à l'apprentissage de la pensée historique, et quelques-unes apparaissent confuses.
Tous droits réservés @ Revue des sciences de l’éducation, 2017
Ce document est protégé par la loi sur le droit d'auteur. L'utilisation des services d’Érudit (y compris la reproduction) est assujettie à sa politique d'utilisation que vous pouvez consulter en ligne.

https://apropos.erudit.org/fr/usagers/politique-dutilisation/ 


\section{Le programme d'Histoire et éducation à la citoyenneté et l'apprentissage de la pensée historique : une analyse lexicométrique}

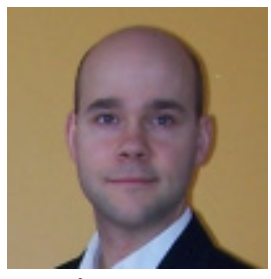

Daniel Moreau

Professeur

Université du Québec en Abitibi-Témiscamingue

RÉSUMÉ - Cet article poursuit dans le sillage d'une analyse conceptuelle réalisée antérieurement. Dans cette analyse, nous interrogions le rapport des programmes d'histoire publiés au Québec depuis la réforme curriculaire entamée au début des années 1980 à l'apprentissage de la pensée historique. Cet article a pour objectif d'éclaircir ces divergences par une analyse lexicométrique du programme d'Histoire et éducation à la citoyenneté. Pour ce faire, une analyse factorielle des correspondances a été réalisée de manière à mettre au jour des traits structuraux spécifiques. Ces derniers rejoignent certaines propriétés associées à l'apprentissage de la pensée historique, et quelques-unes apparaissent confuses.

MOTS-CLÉS - programme d'études, enseignement et apprentissage de l'histoire, pensée historique, analyse lexicométrique, Histoire et éducation à la citoyenneté.

Les résultats présentés dans cet article décrivent une analyse conceptuelle amorcée précédemment (Moreau, 2012). Cette analyse interrogeait le rapport qu'entretiennent les programmes d'histoire publiés au Québec depuis la réforme curriculaire entamée au début des années 1980 à l'apprentissage de la pensée historique chez les élèves du secondaire. L'objectif de cet article est de décrire, à partir d'une démarche lexicométrique exploratoire, la manière dont le programme d'Histoire et éducation à la citoyenneté (Gouvernement du Québec, 2007) intègre la question de l'apprentissage de la pensée historique. Le corpus textuel étudié a été soumis à une opération statistique, à savoir l'analyse factorielle des correspondances. Cette analyse a permis d'identifier certains des traits structuraux spécifiques de l'apprentissage de la pensée historique dans le programme (Gouvernement du Québec, 2007). Cependant, les résultats obtenus étant nuancés, ils invitent à poursuivre les recherches concernant l'enseignement de la pensée historique chez les jeunes. 


\section{Introduction et problématique}

Le programme d'Histoire et éducation à la citoyenneté (Gouvernement du Québec, 2007) de deuxième cycle du secondaire a suscité de nombreux débats au Québec depuis la publication de sa première mouture en 2006. Remplaçant le programme d'Histoire du Québec et du Canada (Gouvernement du Québec, 1982), celle-ci a coulé d'abord dans les médias à travers la plume de Robitaille, qui l'a décrite comme une histoire moins conflictuelle, moins politique et davantage plurielle, accordant une place plus importante aux autochtones et aux groupes non francophones (Robitaille, 2006, p.A-1). Les réactions dans les médias ont été par la suite rapides, et parfois très dures, notamment celle de Bombardier: Les petits manuels à la gloire de la souveraineté paraissent inoffensifs devant cette réécriture historique. Dans le premier cas, on est naïf; dans le second, on est stalinien. (Bombardier, 2006, p.B -5).

Le débat s'est poursuivi au sein de la communauté scientifique - composée de didacticiens et d'historiens - en des termes moins catégoriques, mais autour de positions

analogues. Pour plusieurs, ce programme d'études pose problème parce qu'il évacue certains contenus historiques relatifs à la question nationale. Des contenus dont l'enseignement revêt une importance particulière, puisqu'il s'adresse à des élèves de 14 à 16 ans pour qui c'est généralement la dernière chance d'apprendre l'histoire et le fonctionnement de leur société d'appartenance. Ainsi, pour Bouvier (2007) et la Coalition pour l'histoire qui a pris forme à partir de 2009, cet enseignement - sans récuser une formation à la citoyenneté - doit d'abord consister en une analyse de la question nationale au Québec et au Canada, marquée depuis la Conquête de 1760 par la domination graduelle des Canadiens par les Britanniques. Cette analyse devrait donc mettre davantage l'accent sur les évènements conflictuels, tels l'Acte d'Union de 1840 et les référendums de 1980, 1992 et 1995, ayant ponctué le paysage politique québécois et canadien. Les associations représentant les historiens professionnels ont poursuivi dans ce sillage, sous couvert d'une critique de l'approche par compétences promue par la réforme scolaire entamée depuis le début des années 2000. L'Institut d'histoire de l'Amérique française a souligné l'importance d'acquérir d'abord les connaissances de base, pour développer ultérieurement des compétences historiennes (Bérubé, 2014). Pour sa part, la Fondation Lionel-Groulx (2009, p.38) a dénoncé cette révolution pédagogique, ayant 
détourné l'école de sa mission première : transmettre les faits structurants de l'histoire du Québec.

Pour d'autres didacticiens, la question des contenus est assujettie à une finalité d'éducation à la citoyenneté qui se veut à la fois inclusive, ouverte et critique. Dagenais et Laville (2007) ont souligné les premiers qu'il s'agit à cet égard d'une orientation des programmes d'études de plusieurs pays occidentaux depuis les années 1950. Une orientation fondée sur le principe que l'enseignement de l'histoire ne vise pas l'apprentissage d'un ensemble de connaissances relatives à la question nationale, mais plutôt l'acquisition d'un mode de pensée historique. Selon Éthier, Cardin et Lefrançois (2014), Moisan (2010) et Martineau (2010), cette orientation caractériserait les programmes d'histoire au Québec depuis au moins le début des années 1980. Ce mode de pensée consiste à interroger les réalités sociales dans la perspective du temps pour les expliquer en mobilisant différents concepts et une méthode d'analyse et de critique des sources inspirée de celle des historiens. L'exercice répété de cette méthode concourrait ainsi à l'acquisition d'habiletés intellectuelles, ces dernières pouvant être réinvesties dans l'exercice de la citoyenneté par les élèves.

Sous cet angle, nous avons analysé précédemment (Moreau, 2012) la place de l'apprentissage de ce mode de pensée dans les programmes d'Histoire du Québec et du Canada (Gouvernement du Québec, 1982) et d'Histoire et éducation à la citoyenneté (Gouvernement du Québec, 2007). Notre analyse a révélé des divergences entre ces deux programmes d'études, ce qui a soulevé des interrogations sur la nature de l'apprentissage proposé par ces derniers. Nous avons donc convenu qu'une analyse plus approfondie était nécessaire pour en rendre compte. C'est dans cet esprit que nous proposons une analyse lexicométrique du programme d'Histoire et éducation à la citoyenneté (Gouvernement du Québec, 2007), afin de mettre en lumière, dans une perspective exploratoire, la manière dont il intègre cette question de l'apprentissage de la pensée historique. Est-ce conforme aux définitions que nous retrouvons dans la documentation scientifique? Le cas échéant, le programme exprime-t-il une orientation particulière? Par ailleurs, contribue-t-il effectivement à éclipser la question des contenus, soulevée par ses opposants? Les réponses à ces questions contribueront au débat actuel en plus d'enrichir notre compréhension de la formation intellectuelle visée. Historiquement, une telle formation 
serait au cœur des disciplines scolaires enseignées au secondaire (Chervel, 1998), mais la nature et la place de celle-ci soulèvent des interrogations dans le cas du programme d'Histoire et éducation à la citoyenneté (Gouvernement du Québec, 2007). Mettre en lumière cette formation, sous l'angle de l'apprentissage de la pensée historique, en prenant en compte à la fois les orientations et les contenus de ce programme d'études, permettra d'en faire une interprétation plus juste. Par ailleurs, cette analyse contribuera à une meilleure compréhension de la pensée historique et des modalités présidant à son apprentissage dans le contexte de son enseignement au secondaire au Québec.

Avant de présenter les résultats de cette analyse lexicométrique, nous aborderons, dans un premier temps, la question de l'apprentissage de la pensée historique en classe d'histoire. Nous présenterons également le concept de programme d'études. Dans un deuxième temps, nous décrirons les modalités d'une analyse de contenu lexicométrique permettant, par une analyse factorielle des correspondances, d'identifier des traits structuraux du corpus à l'étude. Ces traits seront décrits dans un troisième temps. Finalement, dans un quatrième temps, ils seront discutés au regard des discours actuels sur le programme d'Histoire et éducation à la citoyenneté (Gouvernement du Québec, 2007).

\section{Contexte théorique}

Nous traiterons dans cette section de l'apprentissage de la pensée historique et du concept de programme d'études. Il s'agira ainsi de préciser d'abord la nature de cet apprentissage à la lumière de la documentation scientifique pour, ensuite, le situer dans le contexte spécifique du programme d'Histoire et éducation à la citoyenneté (Gouvernement du Québec, 2007). Cet exercice permettra de justifier notre démarche d'analyse et de définir les balises conceptuelles nécessaires à la description et à l'interprétation des données.

\section{1 L'apprentissage de la pensée historique}

À l'heure actuelle, il n'existerait pas de définition consensuelle de la pensée historique (Demers, Lefrançois et Éthier, 2010). Nous pourrions ajouter, à fortiori, qu'il n'y a pas de consensus concernant les modalités de son apprentissage. Néanmoins, un bref historique de ce construit permettra d'en circonscrire les grandes lignes. 
L'intérêt pour la pensée historique a émergé dans la documentation scientifique anglophone sur l'enseignement de l'histoire à la fin des années 1950, en GrandeBretagne, dans la foulée des travaux de Peel (1967). Ces derniers, qui donnent le coup d'envoi à la recherche empirique sur l'enseignement de l'histoire (Laville, 2003), expliquent l'apprentissage de ce mode de pensée par la nature de la démarche scientifique et le principe de la balance cognitive entre les fonctions descriptive et explicative des concepts qu'elle met en œuvre (Peel, 1967). Suivant ce sillage, Hallam (1967) puise plutôt aux stades de développement piagétiens pour décrire les difficultés des adolescents de moins de 16 ans à faire preuve de pensée historique, cette dernière exprimant un niveau formel de développement. Les travaux sur la pensée historique se renouvèlent au cours des années 1970 avec l'émergence de ce qui peut être désigné comme seconde école britannique, posant la question de la pensée historique et de son apprentissage sous l'angle des concepts disciplinaires. Ces derniers traduisent l'expertise historienne intervenant dans la production des savoirs historiques et la réalisation de la démarche de résolution de problème en histoire (Lee, 2005; Levstik, 2011; VanSledright et Limon, 2006).

Une telle réflexion sur l'apprentissage de concepts disciplinaires s'est concrétisée à travers deux tentatives de réformes curriculaires britanniques. La première est celle du School's Council History 13-16 project, entreprise en 1972 à 1'Université de Leed (Wineburg, 1996). Elle a débuté avec une soixantaine d'écoles, pour progresser au cours des dix années suivantes, au point de regrouper $20 \%$ de l'ensemble des établissements d'enseignement secondaire (Dawson, 1989). La seconde tentative se nomme Concepts of history and teaching approaches : 7 to 14 (CHATA); réalisée au cours de la première moitié des années 1990, elle cible les concepts disciplinaires de preuve, de causalité et d'explication (Lee et Ashby, 2001; Levstik, 2011). Par ailleurs, cet intérêt pour les concepts disciplinaires a trouvé un écho favorable au Canada avec le Projet de la pensée historique articulé autour de ceux de pertinence historique, de causes et de conséquences, de points de vue historiques, de continuité et de changement, de preuve et de réflexion éthique en histoire (Seixas et Morton, 2012).

Également, les travaux sur l'apprentissage de la pensée historique se sont renouvelés dans la seconde moitié des années 1980, sous l'impulsion de ce qu'il est 
convenu d'appeler la révolution cognitive (Levstik, 2011; VanSledright et Limon, 2006; Wineburg, 1996). La pensée historique est alors définie en fonction des savoirs procéduraux impliqués dans la réalisation de tâches spécifiques (ou heuristiques) relatives à l'analyse documentaire en histoire. Ces tâches concernent la critique et l'identification des sources, la corroboration d'informations à l'aide de sources diverses et la contextualisation d'évènements historiques (VanSledright et Limon, 2006). Notons toutefois que les travaux d'inspiration britannique n'ont pas adopté ce virage procédural, car les concepts disciplinaires sont réputés exprimer en eux-mêmes la dimension opératoire de l'exercice de la pensée historique (Lee, 2005).

Le discours scientifique franco-québécois semble présenter une évolution similaire, avec quelques années de décalage toutefois, alors que, à notre connaissance, c'est avec Laville (1975) qu'apparait cet intérêt pour la pensée historique. Ce construit, dont nous récupèrerons la définition aux fins de notre analyse, est entendu comme une démarche de nature hypothéticodéductive analogue à celle caractérisant le raisonnement scientifique. Cette définition se distingue de la documentation anglophone et de son attachement aux concepts disciplinaires, mais elle récupère le principe d'une démarche à caractère scientifique articulée à des concepts. Cette démarche est enclenchée par l'expression d'interrogations sur le passé, et la pensée historique intervient dans l'analyse d'un problème, la formulation de questions de recherche et d'hypothèses et la validation de ces dernières en fonction des données colligées. C'est sur la base de cette démarche, recensée dans le programme d'Histoire du Québec et du Canada (Gouvernement du Québec, 1982), que Martineau (1997) précise un mode de pensée historique et son apprentissage. Ce mode de pensée est posé en fonction du langage de l'histoire, d'une attitude historienne et d'une méthode de résolution de problème propre à la discipline historique décrivant une culture historienne de pratique. Dans la perspective cognitiviste des travaux sur les savoirs procéduraux, la démarche de résolution de problème est définie comme un processus de traitement de l'information dont l'exercice répété est réputé concourir à une automatisation plus ou moins systématique de l'ordre du réflexe (Martineau, 1997). L'apprentissage de ce mode de pensée, qui doit être une visée de formation intellectuelle explicite, se réalise grâce à des exercices fréquents dans un contexte signifiant, interactif et favorable au transfert et à la métacognition. Demers, 
Lefrançois et Éthier (2010) mentionnent que cette définition de la pensée historique, entendue comme une démarche construite, méthodique et critique, traverse les écrits de plusieurs chercheurs. Selon ces auteurs, le développement de la pensée historique s'opèrerait par la réalisation fréquente d'enquêtes historiques débouchant sur une confrontation des différentes interprétations. Une telle définition pourrait avoir passablement inspiré les auteurs du programme d'Histoire et éducation à la citoyenneté (Gouvernement du Québec, 2007), dont il sera question dans la seconde partie de cette section consacrée au contexte théorique. Toutefois, seule une forme d'analyse de contenu comme une analyse lexicométrique permettra de valider cette hypothèse. Les résultats en seront présentés ultérieurement.

\section{2 Le programme d'Histoire et éducation à la citoyenneté}

Une formation intellectuelle, peu importe la discipline d'enseignement, s'objective dans un programme d'études, et ce dernier définit des expériences d'apprentissage permettant aux enseignants qui en sont responsables de l'accomplir au cours d'une période d'enseignement donnée, généralement - mais pas nécessairement - une année scolaire. Le programme d'Histoire et éducation à la citoyenneté (Gouvernement du Québec, 2007) est enseigné aux premier et deuxième cycles du secondaire. Au premier cycle, il recouvre la géographie et l'histoire générales, tandis qu'au second cycle, il cible ce qu'il est convenu d'appeler l'histoire nationale. C'est de ce dernier cycle qu'il sera question ici, réparti sur deux années scolaires. L'expérience d'apprentissage est chronologique en troisième année du secondaire, tandis qu'elle devient thématique en quatrième année. Toutefois, au moment d'écrire ces lignes, le ministère de l'Éducation, de l'Enseignement supérieur et de la Recherche est en train de procéder à l'évaluation d'un projet pilote d'enseignement de l'histoire, proposant un enseignement chronologique réparti sur ces deux années scolaires. Il pourrait éventuellement le mettre en œuvre à la grandeur du Québec au cours des prochaines années.

Les expériences d'apprentissages proposées par un programme d'études pour réaliser une formation intellectuelle sont balisées par un certain nombre de contenus d'apprentissage, d'objectifs et de démarches d'apprentissage et d'évaluation (Glatthorn, Boschee et Whitehead, 2006). C'est à ce niveau que les débats entourant le programme 
d'Histoire et éducation à la citoyenneté (Gouvernement du Québec, 2007) ont été les plus vifs. Quels contenus pour quelle formation? Ces contenus, trop nationalistes ou trop peu, compromettent-ils la valeur de la formation intellectuelle visée? Sarra-Bournet (2016), qui soulève l'hypothèse d'une crise du cadre national, s'interroge à l'égard de cette formation intellectuelle visée par l'enseignement de l'histoire. Il rappelle la pertinence de l'espace national comme système d'intelligibilité et de contextualisation d'un florilège de réalités sociales, et il spécifie que celui-ci est signifiant d'abord et avant tout parce qu'il est politique (Sarra-Bournet, 2016, p.173). En outre, ce programme d'études témoignerait d'un déclin des savoirs disciplinaires et d'un fétichisme des compétences, attribuable à l'influence grandissante des spécialistes des sciences de l'éducation - notamment des didacticiens - sur la production des programmes d'études. Des programmes qui appréhenderaient les démarches d'enseignement-apprentissage sous l'angle du processus de construction des connaissances par l'élève, et non sous celui de leur transmission par l'enseignant.

Les défenseurs de ce programme d'études ont plutôt invoqué la nécessité d'une prise de distance à l'égard du cadre national pour développer les savoir-faire associés à la pensée historique dans un contexte de résolution de problème. C'est le cas de Dagenais et Laville (2014, p.186) qui indiquent que la formation de citoyens critiques, capables d'interpréter les faits et de s'engager dans une participation sociale réfléchie requiert un changement des contenus scolaires et des pratiques d'enseignement. Ces contenus plus étroitement associés à la pensée historique, comme Martineau $(1997,2010)$ a défini celleci, sont présents dans les programmes d'histoire au Québec par la méthode historique, le langage de l'histoire (thèmes, concepts, repères culturels, connaissances) et les attitudes fondant son exercice. À cet égard, le programme d'Histoire et éducation à la citoyenneté (Gouvernement du Québec, 2007, p.1) spécifie que L'apprentissage de l'histoire favorise, par ailleurs, le développement d'une démarche intellectuelle, d'un langage et d'attitudes qui rendent possible l'appropriation graduelle d'un mode de pensée historique. Dans les pays anglophones, les contenus associés à l'apprentissage de la pensée historique vont s'articuler davantage aux concepts disciplinaires mentionnés précédemment. Au Canada anglophone, notamment, les programmes d'études sont plus susceptibles de référer aux concepts de pertinence historique, de causes et de 
conséquences, de points de vue historiques, de continuité et de changement, de preuve et de réflexion éthique en histoire.

Ces orientations curriculaires ont été initialement circonscrites par les auteurs du rapport Lacoursière (Groupe de travail sur l'enseignement de l'histoire, 1996), soulevant pour la première fois la question de la pensée historique dans la documentation officielle. Les objectifs d'apprentissage y sont alors posés sous l'angle de la formation intellectuelle et sociale de l'enseignement de l'histoire. Cette formation vise à la fois l'acquisition d'une démarche d'analyse des réalités sociales et des connaissances fondant la participation de tous aux débats et à la délibération démocratique. La démarche intellectuelle associée à la pensée historique est alors posée en tant que processus de résolution de problème. Ces orientations curriculaires seraient, par ailleurs, conformes au discours de la recherche sur l'enseignement de l'histoire (Martineau, 2006).

Les compétences disciplinaires et la démarche d'apprentissage définies par le programme d'Histoire et éducation à la citoyenneté (Gouvernement du Québec, 2007) poursuivent dans ce sillage. La pensée historique est articulée autour de trois compétences disciplinaires: 1) interroger les réalités sociales dans une perspective historique, 2) interpréter à l'aide de la méthode historique, 3) consolider l'exercice de sa citoyenneté à l'aide de l'histoire (Gouvernement du Québec, 2007). Ces trois compétences décrivent une démarche d'apprentissage procédant par une phase de problématisation (compétence 1), suivie par la mise en œuvre de la méthode historique (compétence 2) et complétée par l'interprétation de réalités sociales dans la perspective d'une citoyenneté critique et participative à exercer (compétence 3) (Cardin, 2014; Moisan, 2010). Une réalité sociale désigne les actions humaines pouvant être analysées d'un point de vue social, culturel, économique, politique et territorial. L'interrogation des réalités sociales consiste à placer l'élève en situation d'apprentissage en formulant des questions qui se prêtent à l'analyse à l'aide d'un concept. Par exemple, le concept d'industrialisation apporte un éclairage spécifique sur un contexte historique marqué par la concentration des moyens de production en milieux urbains et la création d'une classe ouvrière œuvrant dans des conditions difficiles. Les interrogations soulevées à l'égard de ces conditions pavent la voie au développement de la deuxième compétence, soit la mise en œuvre de la méthode historique à l'aide de concepts - celui d'industrialisation dans ce 
cas-ci - dans l'interprétation de réalités sociales. Cette étape consiste à collecter et à traiter des informations pour répondre aux questions initiales (Cardin, 2014). La troisième compétence exprime une étape de synthèse et d'intégration des apprentissages. À cette étape, l'élève sera en mesure de mobiliser d'autres concepts et des connaissances au regard d'un enjeu du présent, qu'il s'agit d'interpréter, ou vis-à-vis duquel il sera invité à prendre position. La pensée historique, en raison de l'attitude sous-tendant son exercice, est réputée favoriser la prise de conscience de la démocratie dans la durée pour susciter la participation démocratique (Martineau et Laville, 1998). Le langage et la méthode historiques fournissent au citoyen les instruments réutilisables de cette réflexion pour comprendre les différentes réalités sociales et pour agir de manière consciente et réfléchie (Dagenais et Laville, 2007).

Cette démarche d'apprentissage doit être régulée par l'enseignant qui planifie et met en œuvre des situations d'apprentissage et d'évaluation permettant aux élèves de s'exercer aux compétences disciplinaires. En ce sens, un programme d'études impose des balises aux expériences d'apprentissage, mais la manière dont celles-ci seront effectives dépend de la transposition didactique effectuée par les enseignants. Cette transposition n'est toutefois pas l'objet de la présente analyse. Ce programme d'études apporte des précisions conformes aux six critères définissant, selon Martineau (1997), l'apprentissage de la pensée historique. D'abord, la formation intellectuelle visée à travers l'apprentissage de la pensée historique y est explicitement mentionnée. Ensuite, le contexte d'apprentissage doit être signifiant pour l'élève, dans la mesure où les problèmes à résoudre concernent des enjeux de société importants qui ne sont pas étrangers aux élèves. On y souligne aussi que le contexte doit être interactif, car l'apprentissage procède par l'élaboration et la confrontation des interprétations historiques, conformément à l'esprit de la troisième compétence disciplinaire. La question de la métacognition et celle $\mathrm{du}$ transfert apparaissent également dans ce programme, invitant les enseignants à intervenir au niveau des stratégies métacognitives de leurs élèves pour les aider à prendre conscience de leur démarche (Gouvernement du Québec, 2007). Finalement, comme nous l'avons constaté antérieurement (Moreau, 2012), ce programme d'études souligne l'importance de la fréquence de l'exercice de la pensée historique contribuant au développement des habiletés citoyennes visées. Au terme de ces considérations 
conceptuelles, nous pouvons avancer l'hypothèse que l'apprentissage de la pensée historique est une propriété structurante des orientations et des contenus du programme d'Histoire et éducation à la citoyenneté (Gouvernement du Québec, 2007). En somme, ce programme définit une démarche d'apprentissage fondée sur la résolution de problème autour de contenus visant le développement de trois compétences disciplinaires. Nous pouvons néanmoins nous interroger sur l'articulation de ces éléments au regard d'une visée de formation intellectuelle. Seule une analyse de contenu, qui suivra les modalités d'une approche lexicométrique présentées dans la prochaine partie, permettra de rendre compte de cette articulation, à travers une description des éléments structurants de l'apprentissage d'un mode de pensée historique.

\section{Une analyse lexicométrique}

Le rapport du programme d'Histoire et éducation à la citoyenneté (Gouvernement du Québec, 2007) à l'apprentissage de la pensée historique sera éclairci par une analyse lexicométrique. Celle-ci consiste, dans le cadre d'une démarche exploratoire, à soumettre un corpus textuel à des opérations statistiques (Lebart et Salem, 1994), à savoir l'analyse factorielle des correspondances. Cette dernière génère une représentation multidimensionnelle des éléments de ce corpus sous la forme d'un nuage de points se prêtant à l'identification de traits structuraux. En outre, cette opération permet, à des fins interprétatives, de consulter le contexte d'usage des formes lexicales - ou concordances jugées structurantes. En ce sens, une approche lexicométrique révèle une métainformation exceptionnelle (Lebart et Salem, 1994) qui sera utile à la compréhension du programme d'Histoire et éducation à la citoyenneté (Gouvernement du Québec, 2007). Toutefois, cette approche à l'analyse de contenu doit être comprise comme une description préliminaire du corpus constitué et non comme une analyse approfondie du construit de pensée historique dans la documentation officielle. Il pourrait arriver, en raison de la réduction des données impliquée par cette approche, qu'aucun élément théorique présenté précédemment ne puisse être recensé. Cela ne signifie pas pour autant que ce construit ne s'y retrouve pas; c'est simplement que les mots le décrivant sont moins fréquents. 
Avant d'aborder les modalités d'analyse et d'interprétation des données, nous allons présenter successivement le corpus constitué à cette fin et l'instrumentation sollicitée pour la réaliser.

\section{1 Constitution du corpus (variable-sujet)}

Le corpus textuel analysé a été constitué à partir du programme d'Histoire et éducation à la citoyenneté (Gouvernement du Québec, 2007), en fonction des critères que donnent Glatthorn, Boschee et Whitehead (2006) du concept de programme d'études (course of study). Pour chacune des disciplines scolaires, un programme d'études décline les visées globales d'un curriculum en quatre critères d'analyse: des contenus, des objectifs d'apprentissages, des approches pédagogiques et des modalités d'évaluation. Ces quatre critères ont présidé à la constitution d'autant de corpus de textes qui ont été soumis à l'analyse en tant que variables-sujets. Le regroupement des portions de texte pour chacun de ces critères a d'abord été réalisé à la suite de la transcription systématique de l'ensemble de ce programme d'études. Une lecture fine a ensuite permis de sélectionner les extraits relatifs à l'un ou l'autre de ces critères. Le tableau 1 présente les pages sélectionnées et écartées dans la constitution du corpus. Un coup d'œil sur celui-ci permet également de constater la faiblesse des données concernant l'évaluation. Toutefois, les tableaux et les schémas - passablement nombreux dans ce programme d'études - n'ont pas été pris en compte. Ce choix explique également pourquoi le Cadre d'évaluation des apprentissages (Gouvernement du Québec, 2011a) et la Progression des apprentissages (Gouvernement du Québec, 2011b) - où les tableaux y sont dominants - relatifs à ce programme d'études n'ont pas été soumis à l'analyse. En outre, la transcription a été effectuée conformément aux instructions de balisage du logiciel sollicité pour l'analyse.

\section{2 Instrumentation}

Nous avons eu recours au logiciel DTM-Vic pour traiter les données retranscrites convenant à une démarche lexicométrique exploratoire. Il existe d'autres logiciels autorisant le recours à ces dernières, mais le principal argument en faveur de celui-ci tient en la possibilité de soumettre les données à un processus de validation par rééchantillonnage (bootstrap) attestant de leur stabilité, sur lequel nous reviendrons plus 
loin. Ce logiciel permet de réaliser une opération de segmentation, préalable à l'analyse factorielle des correspondances, dont il sera question dans la prochaine sous-section. La segmentation est un traitement automatisé consistant à découper le texte du corpus en formes lexicales (mots). Ce traitement est nécessaire, car les méthodes de la statistique lexicale ne peuvent être appliquées que sur des formes générales d'un même type (Lebart, Piron et Steiner, 2003; Lebart et Salem, 1994). Il a été effectué suivant le protocole d'indexation minimale élaboré par le laboratoire de lexicologie politique de l'École normale supérieure de Saint-Cloud, définissant ces formes comme un ensemble de caractères entre des espaces blancs et des signes de ponctuation (Lafon et Salem, 1983). Ce traitement est automatisé pour préserver la base de données de l'arbitraire du chercheur, toujours susceptible d'intervenir lorsque la signification des mots lui apparait équivoque ou discutable.

Cette autonomisation n'implique pas pour autant une absence totale d'intervention du chercheur à l'endroit de la base de données, mais celle-ci est limitée aux processus de lemmatisation et de désambigüisation. Après une première segmentation, nous avons ainsi soumis l'ensemble des formes distinctes du corpus (son vocabulaire) à un examen minutieux pour regrouper les formes appartenant à un même vocable (Muller, 1967). Par exemple, les formes baie et james et l'ensemble des noms propres sont inintelligibles s'ils sont présentés séparément; aussi ont-ils été regroupés en les faisant précéder d'un dièse (\#baiejames). À la suite de cet examen, nous avons procédé en fonction d'une norme de lemmatisation peu interventionniste pour garantir la richesse des données par le recours, autant que possible, à la désambigüisation. La désambigüisation consiste à séparer les formes homographes relevant de vocables différents ou d'une même origine étymologique. Ainsi, les formes acte, fait ou compétence se sont vues accolées d'un \#, selon le contexte de leur utilisation dans le discours, pour distinguer l'évènement constitutionnel (tel l'Acte de Québec), le fait historique de l'action ou le champ d'action gouvernemental (par exemple : de compétence fédérale) du développement des sujets apprenants (en référence aux compétences disciplinaires). Certaines formes importantes, mais toujours un peu équivoques, tels histoire ou passé, ont volontairement été soustraites de cette désambigüisation dans la mesure où leur signification était suffisamment éclairée par leur contexte d'actualisation. À cet égard, l'application d'une 
norme lexicologique consiste à apprêter le corpus aux fins d'analyse, et non pas à entamer cette analyse (Lebart et Salem, 1994; Muller, 1967). L'équivocité irréductible des vocables, liée à leur contexte d'actualisation, est la raison même de l'analyse lexicométrique, car elle est la cause de la variabilité/invariabilité recherchée au sein des discours.

\section{3 Méthode d'analyse des données}

L'analyse factorielle consiste à produire une représentation multidimensionnelle des éléments du corpus préalablement inscrits dans le tableau lexical entier où est inscrit l'ensemble des formes-lignes et des sujets-colonnes. Cette représentation graphique est générée par la confrontation de ces deux partitions de données pour identifier des variations par catégories (Lebart et Salem, 1994). Ces variations définissent des proximités qui sont calculées en fonction des distances algébriques entre l'ensemble des éléments des lignes et des colonnes, celles de l'une de ces dimensions étant établies statistiquement par celles de l'autre. Par ailleurs, mentionnons que ce n'est pas l'ensemble du vocabulaire du corpus qui a été soumis à l'analyse, mais une portion de celui-ci, délimitée par le choix d'un seuil de fréquence. L'identification et l'interprétation de profils lexicaux ne sont possibles que si les formes apparaissent à une certaine fréquence (Lebart et Salem, 1994). Dans le contexte de cette recherche, nous n'avons soumis à l'analyse que les formes lexicales dont la fréquence est égale ou supérieure à dix.

C'est sous la forme d'un nuage de points que les éléments du corpus sont représentés et traversés d'axes factoriels permettant d'interpréter leurs positions respectives. Au regard de ces axes, le logiciel fournit les valeurs propres - oscillant entre 0 et 1 - exprimant leur inertie respective au sein du nuage de points. C'est cette inertie, exprimant leur valeur explicative à l'endroit de la variance totale, qui permet d'interpréter la position des éléments du corpus. Cela revient à reconnaître une confiance accrue aux premiers axes, d'inertie plus élevée, car plus la valeur propre est proche de 1, meilleure est la qualité de la représentation barycentrique (Lebart et Salem, 1994). Par ailleurs, outre l'inertie, la qualité de la représentation graphique peut varier en fonction d'un effet de projection que la représentation en deux dimensions ne permet pas d'illustrer, trahissant ainsi la position 
réelle de certaines formes lexicales dans le nuage de points. L'interprétation a été effectuée pour les trois axes générés, en procédant, d'abord, par l'identification des formes lexicales ayant une inertie élevée. Cette interprétation a été complétée par l'examen des concordances relatives à ces formes lexicales structurantes, rendant compte de leur contexte d'actualisation dans un corpus.

En complément à l'analyse factorielle des correspondances, nous avons eu recours à une méthode de validation des données par rééchantillonnage (bootstrap). Celle-ci permet de s'assurer de la stabilité des axes à la lumière de celle des formes structurantes dans le nuage de points (Lebart, Piron et Steiner, 2003). Cette opération est nécessaire, car il se peut qu'une part de la variance fluctue en fonction d'une modification de l'échantillon (Lebart, Piron et Steiner, 2003). La validation permet ainsi de critiquer la validité des résultats selon que les formes structurantes s'avèrent stables ou non. La validation par rééchantillonnage utilisée consiste à perturber les données par l'ajout de formes lexicales sélectionnées, en tant que variables supplémentaires, à travers l'effectuation de 25 tirages avec remise (Lebart, Piron et Steiner, 2003). En principe, si les formes lexicales structurantes sont stables, elles apparaitront dans le plan factoriel passablement au même endroit qu'à l'origine. Si ce n'est pas le cas, le logiciel tracera une zone de projection. Celle-ci, selon sa taille, permettra alors de porter un jugement qualitatif sur son niveau de stabilité.

Les données présentées dans la prochaine section ont été interprétées à la lumière des contenus, des objectifs d'apprentissages et des approches pédagogiques promues pour l'apprentissage de la pensée historique. La question de l'évaluation a été retenue dans la constitution de la base de données, car elle est présente dans ce programme d'études. Son interprétation est toutefois valide à l'intérieur du discours commun de celui-ci sur l'enseignement de l'histoire mais elle demeure sujette à caution à cause de la minceur des extraits lui étant associés. En outre, elle est traitée de manière plus opératoire dans le Cadre d'évaluation des apprentissages (Gouvernement du Québec, 2011a). Une interprétation valide de l'évaluation de l'apprentissage de la pensée historique exigerait de prendre en compte ce dernier document. 


\section{Résultats}

Le corpus soumis à l'analyse présente un faible niveau de diversification du lexique, le taux avoisinant les $13 \%$. En comparaison, les textes syndicaux et les questionnaires socioéconomiques analysés par Lebart et Salem (1994) affichent respectivement des taux de $16 \%$ et $8 \%$. L'analyse factorielle des correspondances a généré trois axes expliquant respectivement $54,33 \%, 32,88 \%$ et $12,79 \%$ de l'inertie. Elle est représentée par le plan factoriel de la figure 1. Chacun de ces axes sera décrit au cours des prochaines soussections en présentant d'abord le ou les critères concernés à cause de sa ou leur contribution significative, ainsi que les formes les plus structurantes. Celles-ci seront ensuite interprétées à la lumière des concordances, c'est-à-dire de leur contexte d'apparition dans le discours analysé.

\subsection{Axe 1 : l'opposition entre les contenus et la démarche d'apprentissage}

Un premier coup d'œil sur cet axe permet de constater un rapport d'opposition entre les contenus à enseigner (portion négative) et les approches pédagogiques (portion positive), principalement. Comme le montre le tableau 2, le critère relevant des approches pédagogiques contribue le plus fortement à l'inertie de cet axe, à hauteur de 50,3\%. C'est ce qui explique que les formes les plus structurantes gravitent dans cette zone (figure 2). Situés également dans la même portion de cet axe, les critères relatifs aux objectifs et à l'évaluation ne comptent respectivement que pour $8,3 \%$ et $4,4 \%$ de l'inertie. Ces formes structurantes, illustrées par la figure 2, concernent la démarche d'apprentissage de l'élève : élève, démarche, enseignant, documents, évaluation, repérer, information, sa, inscrire, données, réalisation, compétence.

Ces formes structurantes expriment ce qui est attendu de l'enseignant en termes d'encadrement et de support à la démarche d'apprentissage. Ainsi, à plusieurs reprises, il est spécifié que L'enseignant fournit à l'élève des outils d'évaluation de sa démarche, et qu'il encadre la démarche de l'élève (Gouvernement du Québec, 2007, p.16, p.21, p.27). Les formes documents, données et réalisation décrivent des propriétés fondamentales de cette démarche qui consiste à travailler avec des documents variés et mettre en œuvre des techniques spécifiques. La validation des formes démarche, enseignant et documents donne lieu à des ellipses de confiance exprimant une relative instabilité, tout comme celle 
de la forme réalisation, qui peut être considérée comme instable. Seule la forme élève apparait relativement stable. D'ailleurs, l'élève doit, selon le programme, juger de la pertinence et de la validité des documents pour établir des liens entre le passé et le présent et élaborer une opinion fondée. À l'égard des documents, l'élève doit développer la maitrise de techniques par leur utilisation fréquente. Ces techniques consistent ainsi à repérer et à inscrire (oblitérée par la première) de l'information (oblitérée par élève) et des données, en lien avec la réalisation de lignes du temps, l'interprétation et la réalisation de cartes, de tableaux à entrées multiples et de diagrammes. La stabilité des formes repérer et inscrire est indiscutable, alors que leur validation ne génère pas de zones de projection perceptibles. Ces comportements attendus de repérage et d'inscription d'informations expriment donc une signification très précise. Inversement, les formes information et données sont les plus instables de ce plan factoriel, comme en témoignent leurs ellipses de confiance. Cela indique que leur signification varie selon le contexte. L'examen des concordances indique que la forme évaluation apparait fréquemment avec celles de situations et d'apprentissage. Toutefois, ces dernières ne se s'étant pas avérées structurantes, nous interprétons la signification de cette forme principalement en fonction du contexte où elle apparait de manière exclusive. C'est le cas lorsqu'il est question du contexte et des critères d'évaluation visant à brosser un portrait du développement de chacune des trois compétences disciplinaires. À cet égard, il est demandé à l'enseignant de fournir à l'élève des outils d'évaluation de sa démarche (Gouvernement du Québec, 2007, p.16, p.21, p.27). Cette forme apparait, à la lumière de sa zone de projection, relativement stable.

À l'autre extrémité, le critère de contenus représente $36,9 \%$ de cette inertie. Celle-ci repose sur la contribution de plusieurs formes structurantes, dont très peu, cependant se distinguent du lot: Québec et pouvoir. Elles peuvent être considérées comme stables à la lumière de leurs zones de projection respectives limitées. Les concordances révèlent que la forme Québec est le plus fréquemment utilisée en lien avec le territoire, l'histoire et la société québécoise actuelle. Le Québec est ainsi régulièrement associé aux traces humaines qui meublent son territoire et qui attestent de la présence des populations amérindiennes et coloniales. Le Québec, surtout apparaît comme une province témoignant de mutations sociales, culturelles et économiques qui accompagnent 
la création de la fédération canadienne. La forme pouvoir renvoie le plus fréquemment aux luttes politiques menées par les Canadiens entre la Conquête de 1759 et les rébellions des Patriotes en 1837. La fréquence élevée de cette forme $(n=44)$, comparable à celle de Québec $(n=55)$, est toutefois attribuable au thème Pouvoir et pouvoirs qui lui est consacré, où elle apparait à 26 reprises. Ce thème invite les élèves à réfléchir sur le concept de pouvoir et sur les groupes d'influence qui l'exercent.

\subsection{Axe 2 : les objectifs d'apprentissage}

Le deuxième axe (vertical) représenté par la figure 3 est davantage structuré par le critère d'objectifs (portion positive), expliquant $64,5 \%$ de son inertie. Comparativement, le critère relevant des approches pédagogiques d'apprentissage dans la portion négative de cet axe en exprime $26,5 \%$. Également situés dans cette portion, les critères relatifs aux contenus et à l'évaluation ne comptent respectivement que pour $7,1 \%$ et $1,9 \%$ de l'inertie de cet axe. Les formes structurantes, présentées dans le tableau 3, décrivent les objectifs visés par ce programme d'études: programme, leur, ils, rencontrées, citoyenneté, réalités, lorsqu, reconnaître, comprendre, sociales, exercice, difficultés. La forme programme réfère aux compétences disciplinaires et transversales du programme d'Histoire et éducation à la citoyenneté (Gouvernement du Québec, 2007), notamment dans la perspective d'établir des liens avec les contenus de formation, les domaines généraux de formation et d'autres programmes d'études, dont ceux du premier cycle. La validation de cette forme donne lieu à une zone de projection limitée exprimant une certaine stabilité. Les formes leur et ils (oblitérée par la première) désignent principalement les élèves, concernés par le développement des compétences. À la lumière de leurs ellipses de confiance, ces formes apparaissent stables, encore une fois. Nous associons également la forme lorsqu - d'une stabilité relative - aux élèves, mais en lien avec une intention d'agir. Cette intention d'agir concerne à la fois l'interrogation et l'interprétation des réalités sociales, le recours au langage de l'histoire et de la cartographie, l'empathie historique (point de vue des acteurs historiques) et la participation aux débats.

Les formes difficultés et rencontrées apparaissent souvent ensemble, généralement dans le contexte de la présentation de chacune des compétences disciplinaires. Le 
développement de ces dernières exige que les élèves réfléchissent sur les difficultés qu'ils rencontrent dans la réalisation de la démarche d'apprentissage pour y remédier. Si la validation de la forme rencontrées ne génère pas d'ellipse perceptible, il en va autrement pour difficultés dont la ligne épaisse de validation indique une relative instabilité. Les formes exercice et citoyenneté expriment l'une des finalités premières de l'enseignement de l'histoire, celle d'apprendre à exercer sa citoyenneté de manière ouverte et éclairée, dans le respect des principes et des valeurs démocratiques. La validation de ces formes donne toutefois lieu à des zones de projection d'une certaine envergure, comparables à celle de la forme difficultés, considérée comme relativement instable. Les formes réalités, sociales, reconnaître et comprendre (oblitérée par reconnaître) décrivent l'objet de savoir concerné et la nature de l'activité intellectuelle à développer chez l'élève. Les concordances indiquent que les réalités sociales relèvent à la fois du présent et du passé et sont étroitement associées à la vie démocratique, en référence à des faits historiques, leurs origines, leur contexte et les acteurs historiques (point de vue, croyances, attitudes, valeurs et intérêts). La compréhension visée concerne l'ensemble de ces aspects des réalités sociales dans une perspective historique, en lien avec les enjeux du présent, la démocratie et ses institutions. Les zones de projection pour les formes réalités et sociales sont d'envergure limitée, ce qui exprime leur stabilité. La validation des formes comprendre et reconnaître donne lieu à des ellipses de confiance plus grandes, traduisant une relative instabilité pour cette première forme structurante : et une relative stabilité pour la seconde. La forme reconnaître désigne trois contextes: 1) celui de la reconnaissance, par les élèves, de leurs forces et de leurs faiblesses dans le développement d'un regard critique sur leur démarche d'apprentissage; 2) celui des identités en développement et 3) celui de l'importance de la prise de parole, incarnant l'action transformative au cours de l'histoire.

À l'autre extrémité de ce deuxième axe, nous retrouvons sensiblement les mêmes formes associées aux approches pédagogiques d'apprentissage (tableau 3), c'est-à-dire enseignant, évaluation, réalisation, repérer, élève, documents et inscrire. À cette liste de formes s'ajoutent tâches et situations (oblitérée par la première forme), dont la validation est représentée par la figure 3. Ces formes structurantes précisent la signification de la démarche d'apprentissage qui se concrétise à travers des situations d'apprentissage et 
d'évaluation impliquant variablement des scénarios, des tâches, des documents (Gouvernement du Québec, 2007, p.8). La validation de cette forme structurante donne lieu à une zone de projection (figure 3) qui exprime une relative instabilité. Ces situations garantissent le développement des compétences disciplinaires visées en définissant un cadre pour l'enseignement des contenus de formation. Ces contenus sont traités à travers la réalisation de tâches qui permettent d'interroger le présent tout en orientant le questionnement des élèves vers le passé (Gouvernement du Québec, 2007, p.8). La nature de ces tâches est variée. Elle peut consister à sélectionner des documents, évaluer différents points de vue d'acteurs ou de témoins de la réalité sociale, comparer des sociétés entre elles, déterminer des liens de causalité, etc. (Gouvernement du Québec, 2007, p.9). Cette imprécision se traduit par une instabilité avérée, exprimée par l'importante ellipse de confiance générée par la validation de cette forme (figure 3).

\subsection{Axe 3 : l'évaluation}

Représenté par la figure 4, le troisième axe (vertical) compte pour 12,79\% de l'inertie totale. Il est structuré par le critère d'évaluation (portion négative), expliquant $91,5 \%$ de la variance. Il exprime donc la spécificité de ce discours, distinct cette fois du critère d'approche pédagogique d'apprentissage - comptant pour 8,4\% de l'inertie - auquel il est opposé. Les formes structurantes, inscrites dans le tableau 4, décrivent les modalités de l'évaluation des apprentissages: évaluation, composantes, compétences, analyse, année, critique, fin, situation, cadre, compte, apprentissage, permet, compétence, historiques. La forme évaluation a déjà validée pour le premier axe et s'est avérée relativement stable. Elle désigne le contexte de la démarche d'évaluation consistant à fournir à l'élève des outils d'évaluation de sa démarche et à rendre compte du développement des compétences.

La forme composantes concerne celles décrivant chacune des compétences disciplinaires sur lesquelles l'enseignant doit fonder l'instrumentation de l'évaluation. Les formes compétences et compétence correspondent aux objectifs et aux contenus de formation de ce programme d'études. Elles se distinguent cependant par leur rapport à la pratique d'enseignement. Si les compétences réfèrent plus largement à ces objectifs et ces contenus, la compétence (au singulier) est plus étroitement associée à l'intervention de 
l'enseignant. Il en est de même pour la forme situation qui décrit une attente spécifique à l'égard de l'enseignant en contexte d'évaluation en termes de tâches proposées aux élèves et d'observations. Les formes cadre (oblitérée par compétences), compte (oblitérée par historiques), permet et apprentissage indiquent à cet égard que la situation d'évaluation représente un contexte spécifique d'intervention contribuant à soutenir le développement des compétences. Les formes année et fin (oblitérée par situation) désignent la durée de la période d'évaluation et les attentes au terme de celle-ci, que ce soit au cours de l'année scolaire, à la fin de celle-ci ou du cycle d'enseignement. Les formes analyse et critique sont utilisées pour décrire le retour réflexif de l'élève sur sa démarche dans le développement de chacune des compétences disciplinaires. La forme historiques renvoie à l'objet de l'évaluation qui sont des connaissances à mobiliser - avec des concepts et des techniques - dans la réalisation de tâches spécifiques. À l'exception de la forme année, la validation de l'ensemble des formes structurantes a généré d'importantes ellipses de confiance, exprimant leur instabilité. Seules les formes compétence, compétences et apprentissage peuvent être considérées comme relativement instables.

À l'autre extrémité de ce troisième axe, les formes documents, repérer*, sa et inscrire*, indiquées dans le tableau 4, rappellent les modalités de l'approche pédagogique. Nous avons interprété ces résultats précédemment pour le premier axe, hormis pour la forme $s a$ référant notamment à l'élève impliqué dans la démarche d'apprentissage. La stabilité de cette forme apparaît discutable, comme pour la majorité de celles contribuant à cet axe dans cette portion.

\subsection{Les résultats : une synthèse}

Cette analyse lexicométrique a révélé des traits structuraux pour chacun des critères d'analyse du programme d'Histoire et éducation à la citoyenneté (Gouvernement du Québec, 2007). Pour les approches pédagogiques d'apprentissage, ces traits structuraux décrivent des démarches d'apprentissage consistant à travailler avec des documents variés et à mettre en œuvre des techniques spécifiques. Les contenus reposent sur la contribution de plusieurs formes lexicales, dont seulement Québec et pouvoir se distinguent. Les objectifs, au deuxième axe, s'articulent au développement des trois compétences disciplinaires. L'évaluation, quatrième critère, vise à rendre compte du 
développement des compétences à la lumière des composantes de celles-ci. À cet égard, il incombe à l'enseignant de mettre en œuvre des situations d'apprentissage, notamment des tâches contextualisées, devant se traduire par la mobilisation de connaissances historiques par les élèves. Une discussion de ces résultats permettra, dans la partie suivante, de les mettre en perspective à la lumière de l'inertie et de la stabilité des axes. Elle sera également l'occasion d'établir des liens avec les écrits scientifiques.

\section{Discussion}

Cette analyse lexicométrique a permis d'atteindre notre objectif de recherche qui consistait à décrire les traits structuraux de l'apprentissage de la pensée historique dans le programme d'Histoire et éducation à la citoyenneté (Gouvernement du Québec, 2007).

Ces traits sont passablement conformes aux prescrits didactiques présentés précédemment, mais avec quelques nuances qui permettront d'enrichir le débat actuel entourant ce programme d'études.

En tant que critère le plus important en termes d'inertie expliquée, les approches pédagogiques se prêtent à deux types d'interprétation, selon leur degré de conformité aux prescrits didactiques. D'une part, elles rendent compte d'une démarche fondée sur la manipulation de documents variés et la mise en œuvre de techniques spécifiques à l'histoire. L'analyse de documents représente effectivement un prescrit didactique associé à la pensée historique, car elle est nécessaire à la mise en œuvre de la méthode historique relative à la deuxième compétence disciplinaire (Cardin, 2014). En ce sens, conformément aux prescrits didactiques, l'apprentissage de la pensée historique est principalement situé dans le contexte d'un exercice fréquent en classe, favorable au transfert et à la métacognition (Martineau, 1997). Comme l'a constaté Sarra-Bournet (2016), le développement de compétences disciplinaires chez les élèves est au cœur de la démarche d'enseignement, sans référence explicite à un contexte de transmission de connaissances. Les formes lexicales décrivant l'activité des élèves sont d'ailleurs plutôt stables. Cet enseignement consiste à juger de la pertinence et de la validité des documents, pour établir des liens entre le passé et le présent et élaborer une opinion fondée. Nous pourrions établir un lien avec le concept disciplinaire de preuve consistant à appuyer une interprétation sur des documents (Seixas et Morton, 2012). 
D'autre part, l'analyse semble révéler une position équivoque à l'égard de certains éléments de la démarche d'apprentissage. C'est le cas en ce qui concerne sa nature hypothéticodéductive et le rôle de l'enseignant dans sa mise en œuvre, qui n'ont pas été exprimés par les données ou alors, le cas échéant, de manière plus ou moins instable. Également, l'importance et la stabilité des formes relatives aux techniques mobilisées par cette démarche laissent songeur. Elles consistent à repérer et à inscrire des informations et des données dans la réalisation et l'interprétation de lignes du temps, de cartes et de tableaux à entrées multiples et de diagrammes. Certes, la mise en œuvre de la démarche d'apprentissage exige le recours à ces techniques, mais l'apprentissage se résume-t-il au repérage et à l'inscription d'informations? Une analyse plus approfondie de ce programme d'études serait nécessaire. Martineau (1997, 2010) appréhende l'apprentissage en fonction du paradigme du traitement de l'information, mais pour définir une démarche d'apprentissage complexe. À cet égard, Boutonnet (2013) associe un travail de repérage et d'inscription d'informations à un usage partiel de la méthode historique, car il manque à celle-ci une situation-problème pour contribuer au développement d'un esprit critique. Soulignons cependant que ces résultats doivent être situés dans le contexte de notre analyse lexicométrique visant à décrire des traits structuraux. Ils n'autorisent pas à penser que ce programme d'études n'associe pas l'apprentissage de la pensée historique à des situations-problèmes au profit de tâches de cueillette d'information. Ces résultats indiquent simplement que les références à ces dernières sont plus fréquentes et réalisées dans des contextes analogues.

Les données relatives aux objectifs d'apprentissage révèlent des orientations conformes à plusieurs prescrits didactiques, notamment en ce qui concerne l'acquisition d'une attitude historique: cette attitude, à la fois critique et ouverte aux autres interprétations, doit s'exprimer à travers l'interrogation et l'interprétation des réalités sociales (recours au langage de l'histoire, empathie historique), ainsi que la participation aux débats de société (Cardin, 2014; Martineau, 1997). En outre, l'enseignement doit contribuer à développer la compréhension des réalités sociales dans une perspective historique. Ces objectifs sont conformes à ceux définis dans la documentation scientifique sur la pensée historique et reposent sur des formes structurantes relativement stables. La seule exception à cet égard concerne l'exercice de la citoyenneté dont les 
formes structurantes sont apparues relativement instables. Cela signifie que celles-ci sont utilisées dans des contextes différents, ce qui rend plus imprécise leur signification.

La question des savoirs disciplinaires, soulevée par les détracteurs de ce programme d'études, n'est pas particulièrement mise en lumière par cette analyse. Cette dernière n'a pas révélé de savoirs spécifiquement relatifs à l'apprentissage de la pensée historique. L'analyse aurait pu mettre en relief certaines formes exprimant des concepts disciplinaires ou plus simplement des attributs de concepts. Le concept peut être associé à la balance cognitive décrite par Peel (1967), ainsi qu'à la question du transfert (Cardin, 2014). Néanmoins - et à notre grand étonnement - les formes Québec et pouvoir se sont avérées plus structurantes et se sont illustrées de manière stable. Ce résultat permet de mettre en perspective cette critique, rappelée par Sarra-Bournet (2016), voulant que ce programme d'études se trouve à consacrer une marginalisation du cadre national et de la dimension politique dans l'étude des réalités historiques. Cette critique pourrait être juste, mais il faudrait alors la valider en approfondissant davantage l'analyse de contenu de ce programme d'études et en soumettant le programme d'histoire précédent à ce même type d'analyse lexicométrique.

Finalement, le critère relatif à l'évaluation rend compte de pratiques visant à favoriser les rétroactions et à susciter chez l'élève un retour réflexif sur sa démarche d'apprentissage. Sous cet angle, ces résultats rejoignent le caractère métacognitif de l'apprentissage de la pensée historique souligné par Martineau (1997). Cependant, l'axe pour lequel ce critère a été identifié est celui qui explique le plus faible pourcentage d'inertie et s'est avéré globalement instable. Par ailleurs, comme nous l'avons mentionné précédemment, l'interprétation de ce critère doit se faire avec prudence à cause de la minceur des indications apportées par ce programme d'études. Ces résultats invitent à poursuivre l'analyse en prenant en compte le Cadre d'évaluation des apprentissages (Gouvernement du Québec, 2011a) qui lui est associé. 


\section{Conclusion}

Cette recherche avait pour objectif de décrire la manière dont le programme d'Histoire et éducation à la citoyenneté (Gouvernement du Québec, 2007) intègre la question de l'apprentissage de la pensée historique. Pour ce faire, nous avons sollicité les principales propriétés attribuées à ce construit dans la documentation scientifique pour les investir dans une analyse lexicométrique. Cette analyse a permis d'identifier des propriétés structurantes et d'en valider la stabilité. Les résultats nous obligent à conclure de manière très nuancée: seules certaines propriétés attribuées à l'apprentissage de la pensée historique se retrouvent dans ce programme d'études. Ces propriétés relèvent du travail sur documents dans le contexte de situations d'apprentissage impliquant des tâches variées et le développement, chez l'élève, d'un regard critique sur sa démarche. Elles rencontrent celles définies dans les écrits scientifiques, en lien avec la fréquence de l'exercice, le transfert et la métacognition. Cependant, ces critères se sont révélés d'une stabilité discutable. Les objectifs expriment des propriétés structurantes et stables autour de l'interrogation et de l'interprétation des réalités sociales. Toutefois, d'autres propriétés structurantes - plus stables encore - décrivent plutôt une démarche d'apprentissage fondée sur le repérage d'informations qui serait peu favorable à l'apprentissage de la pensée historique.

Cette conclusion invite alors à poursuivre la réflexion sur l'apprentissage de la pensée historique et à la valider par l'analyse de deux objets étroitement associés à l'enseignement de l'histoire : les programmes d'études et les pratiques d'enseignement. À l'endroit des programmes d'études, cette recherche invite d'abord à approfondir l'analyse des contenus de formation, notamment dans le contexte de leur renouvèlement. Ensuite, l'attribution de la pensée historique à ces contenus de formation mériterait d'être interrogée pour en valider les fondements et les limites. Le programme précédent avait été interprété de la sorte par Martineau (1997), mais les critères que ce chercheur en retient s'y trouvent-ils effectivement? La même question peut s'appliquer à l'égard du projet-pilote actuellement en cours. Les écrits scientifiques semblent généralement reconnaître une visée d'apprentissage de la pensée historique aux programmes d'études, sans toutefois s'appuyer sur des analyses systématiques. Que ces derniers affichent une intention explicite en ce sens pour se conformer aux prescrits didactiques ou à toute autre 
critère normatif n'autorise pas à penser pour autant qu'ils le font effectivement. Si tel était le cas, quelle serait la pertinence d'une analyse critique des programmes d'études? Nous retrouvons un peu de ce problème soulevé par Couturier (2013) au regard de la réflexivité en éducation, qui fait l'objet d'une inflation normative, notamment attribuable à une variabilité lexicale. Il nous semble en aller de même pour le construit de pensée. À terme, nous pouvons nous interroger sur la signification concrète de la pensée historique et de son apprentissage non seulement pour ces programmes d'études, mais également pour les enseignants qui les interprètent et les appliquent. Boutonnet (2013) a constaté que plusieurs pratiques consistent à repérer des informations dans des ressources didactiques. Et si c'était cela, la signification concrète de l'apprentissage de la pensée historique pour ces enseignants d'histoire?

\section{English TITLE - The History and Citizenship Education Program and learning historical thinking: a lexicometrical analysis}

SUMMARY: This paper continues along a previous conceptual analysis, questioning Quebec's history programs published since the curricular reform of 1980's in regard to learning historical thinking. It is acknowledged that this learning is woven in these history programs, associated with analysis abilities and active citizenship participation. However, a comparative analysis of the History of Québec and Canada program with the History and Citizenship Education program presently implemented revealed divergences about learning historical thinking. The objective of this paper is to shed some light on these differences by doing a lexicometrical analysis of the latter program. A factorial analysis of correspondence permitted a description of the structural features of this program. Some of these features can be attributed to historical thinking learning, and a few seem to be confuse.

KEY WORDS : history curriculum, history teaching and learning, historical thinking, lexicometrical analysis.

Título - El programa de Historia y educación a la ciudadanía y el aprendizaje del pensamiento histórico: un análisis lexicométrico

RESUMEN - Este artículo continúa un análisis conceptual realizado anteriormente, en el que se cuestionaba la relación entre los programas de historia publicados en Quebec a partir de la reforma curricular iniciada al comienzo de los años 80 y el aprendizaje del pensamiento histórico. En este artículo nos planteamos aclarar estas divergencias a través de un análisis lexicométrico del programa de Historia y educación a la ciudadanía. Para ello se realizó un análisis factorial de correspondencias con el objetivo de actualizar algunos rasgos estructurales específicos. Éstos son similares a algunas propiedades asociadas al aprendizaje del pensamiento histórico, aunque algunos parezcan confusos. 
Palabras Clave - Programa de estudio, enseñanza y aprendizaje de la historia, pensamiento histórico, análisis lexicométrico, Historia y educación a la ciudadanía.

\section{Références}

Bérubé, H. (2014). Réforme des programmes d'histoire : position de l'Institut d'histoire de l'Amérique française (IHAF). Traces, 52(1), 17-20.

Bombardier, D. (2006, 29-30 avril). Les belles histoires des pays d'en hauts. Le Devoir, B5.

Boutonnet, V. (2013). Les ressources didactiques: typologie d'usages en lien avec la méthode historique et l'intervention éducative d'enseignants d'histoire au secondaire (Thèse de doctorat non publiée). Université de Montréal, Montréal.

Bouvier, F. (2007). Quand l'histoire nationale devient problématique au nom de l'éducation à la citoyenneté : phénomène à inverser. Bulletin d'histoire politique, 15(2), 89-106.

Cardin, J. F. (2014). Les programmes de sciences sociales : du pourquoi au comment. Dans M.- A. Éthier, D. Lefrançois et S. Demers (dir.), Faire aimer et apprendre l’histoire et la géographie au primaire et au secondaire (p.75-98). Montréal, Québec : Éditions MultiMondes.

Chervel, A. (1998). La culture scolaire. Une approche historique. Paris, France : Belin.

Couturier, Y. (2013). Critique de la réflexivité (Mais est-ce donc possible?). Phronesis, 2(1), 8-14.

Dagenais, M. et Laville, C. (2007). Le naufrage du projet de programme d'histoire «nationale»: retour sur une occasion manquée accompagné de considérations sur l'éducation historique. Revue d'histoire de l'Amérique française, 60(4), 517-550.

Dagenais, M. et Laville, C. (2014). Débats autour de l'histoire scolaire : caractères singuliers, mais enjeu unique. Bulletin d'histoire politique, 22(3), 185-199.

Dawson, I. (1989). The Schools history project: a study in curriculum development. The history teacher, 22(3), 221-238.

Demers, S., Lefrançois, D. et Éthier, M.- A. (2010). Un aperçu des écrits publiés en français et en anglais depuis 1990 à propos des recherches en didactique sur le développement de la pensée historique au primaire. Dans J.- F. Cardin, M.- A. Éthier 
et A. Meunier (dir.), Histoire, musées et éducation à la citoyenneté (p.213-245). Québec, Québec : Éditions MultiMondes.

Éthier, M.-A., Cardin, J.-F. et Lefrançois, D. (2014). Leur programme et le nôtre. Bulletin d'histoire politique, 22(3), 165-178.

Fondation Lionel-Groulx (2009). La réforme des programmes au secondaire sonne le glas de l'enseignement de l'histoire. Traces, 47(1), 35-39.

Glatthorn, A., Boschee, F. and Whitehead, B. (2006). Curriculum leadership. Development and implementation. Thousand Oaks, California: SAGE publications.

Gouvernement du Québec (1982). Programme d'études. Histoire du Québec et du Canada. $4^{e}$ secondaire. Formation générale et professionnelle. Québec, Québec: ministère de l'Éducation.

Gouvernement du Québec (2007). Programme de formation de l'école québécoise. Enseignement secondaire, deuxième cycle. Québec, Québec : ministère de l'Éducation, du Loisir et du Sport.

Gouvernement du Québec (2011a). Cadre d'évaluation des apprentissages. Histoire et éducation à la citoyenneté - Enseignement secondaire $1^{e r}$ et $2^{e}$ cycle. Québec, Québec : ministère de l'Éducation, du Loisir et du Sport.

Gouvernement du Québec (2011b). Progression des apprentissages. Histoire et éducation à la citoyenneté - Enseignement secondaire $3^{e}$ et $4^{e}$ secondaire. Québec, Québec : ministère de l'Éducation, du Loisir et du Sport.

Groupe de travail sur l'enseignement de l'histoire (1996). Se souvenir et devenir. Rapport du Groupe de travail sur l'enseignement de l'histoire. Québec, Québec: Gouvernement du Québec.

Hallam, R. N. (1967). Logical thinking in history. Educational review, 19, 183-202.

Lafon, P. et Salem, A. (1983). L'inventaire des segments répétés d'un texte. Mots, 6, 161176.

Laville, C. (1975). Psychologie de l'adolescent et enseignement historique : le problème de l'accès à la pensée formelle. Cahiers de Clio, 43-44, 33-42.

Laville, C. (2003). Pour une éducation historique critique, qu'attendre du courant de la conscience historique? Dans N. Tutiaux-Guillon et D. Nourrisson (dir.), Identités, 
mémoires, conscience historique (p.13-25). Saint-Étienne, France: Publications de l’Université de Saint-Étienne.

Lebart, L., Piron, et Steiner, J- F. (2003). Sémiométrie. Paris, France : Dunod Éditeur.

Lebart, L. et Salem, A. (1994). Statistique textuelle. Paris, France : Dunod Éditeur.

Lee, P. J. (2005). Putting principles into practice : understanding history. In S. Donovan and J. Bransford (eds). How students learn. history, mathematics, and science in the Classroom (p.31-78). Washington, District of Colombia: National academies press.

Lee, P. J. and Ashby, R. (2001). empathy, perspective taking and rational understanding. In O. Davis jr., S. Foster et E. Yeager (eds), Historical empathy and perspective taking in the social studies. Boulder, Colorado: Rowman and Littlefield.

Levstik, L. (2011). Learning history. In R. E. Mayer and P. A. Alexander (eds), Handbook of research on learning and instruction (p.108-126). New York, New York: Routledge.

Martineau, R. (1997). L'échec de l'APH à l'école secondaire, contribution à l'élaboration de fondements didactiques pour enseigner l'histoire (Thèse de doctorat non publiée). Université Laval, Sainte-Foy.

Martineau, R. (2006). Le Rapport Lacoursière : une relecture dix ans après. Bulletin d'histoire politique, 14(3), 13-29.

Martineau, R. (2010). Fondements et pratiques de l'enseignement de l'histoire à l'école. Traité de didactique. Québec, Québec : Presses de l'Université du Québec.

Martineau, R. et Laville, C. (1998). L'histoire, voie royale vers la citoyenneté. Vie pédagogique, 110, 35-38.

Moisan, S. (2010). Fondements épistémologiques et représentations sociales d'enseignants d'histoire du secondaire à l'égard de l'enseignement de l'histoire et de la formation citoyenne (Thèse de doctorat non publiée). Université de Montréal, Montréal.

Moreau, D. (2012). Les programmes québécois d'histoire nationale (1982-2007) et l'apprentissage de la pensée historique: vers l'identification d'un problème de conceptualisation. Revue des sciences de l'éducation, 38(2), 397-421.

Muller, C. (1967). La statistique lexicale. Langue française, 2, 30-43. 
Peel, E. A. (1967). Some problems in the psychology of history teaching: historical ideas and concepts. In W. H. Burston and D. Thompson (eds), Studies in the nature and teaching of history (p.159-172). London, United Kingdom: Routledge and Kegan Paul.

Robitaille, A. (2006, 27 avril). Cours d'histoire épurés au secondaire. Québec songe à un enseignement «moins politique», non national et plus «pluriel». Le Devoir, A1 et A8.

Sarra-Bournet, M. (2016). La politique de la mémoire. Bulletin d'histoire politique, 24(2), 167-179.

Seixas, P. and Morton, T. (2012). The big six historical thinking concepts. Toronto, Ontario: Nelson Education.

VanSledright, B. and Limon, M. (2006). Learning and teaching social studies: a review of cognitive research in history and geography. In P. Alexander et P. Winne (eds), Handbook of educational psychology (p.545-570). Mahwah, New Jersey: Erlbaum.

Wineburg, S. S. (1996). The psychology of learning and teaching history. In D.C. Berliner, and R.C. Calfee (eds), Handbook of educational psychology (p. 423-437). New York, New York: Simon and Schuster Macmillan.

\section{Correspondance}

Daniel.Moreau@uqat.ca

\section{Contribution de l'auteur}

Daniel Moreau: $100 \%$

Ce texte a été révisé par Viviane Boucher

Texte reçu le : 7 avril 2016

Version finale reçue le : 22 février 2017

Accepté le : 16 mars 2017 


\section{ANNEXES}

Tableau 1

Constitution de la base de données pour chacun des critères

\begin{tabular}{|c|c|c|}
\hline $\begin{array}{l}\text { Critères } \\
\text { d'analyse }\end{array}$ & $\begin{array}{l}\text { Pages du programme d'Histoire et } \\
\text { éducation à la citoyenneté } \\
\text { SOUMISES à l'analyse. }\end{array}$ & $\begin{array}{l}\text { Pages du programme d'Histoire et } \\
\text { éducation à la citoyenneté } \\
\text { EXCLUES de l'analyse. }\end{array}$ \\
\hline $\begin{array}{l}\text { Objectifs } \\
\text { d'apprentissage }\end{array}$ & $\begin{array}{l}1 \text { à } 7 \\
11 \text { à } 27\end{array}$ & $13,15,16,20,21,26$ et 27 \\
\hline $\begin{array}{l}\text { Démarche } \\
\text { pédagogique }\end{array}$ & $8,9,14,19,25$ & \\
\hline Contenus & $\begin{array}{l}29,31 \text { à } 33,36,39,40,43,44,47, \\
48,51,52,55,56,60,61,66,67, \\
70,71,74,75,79,80,84,87,89, \\
92 \text { à } 95\end{array}$ & $\begin{array}{l}28,30,34,35,37,38,41,42,45, \\
46,49,50,53,54,57 \text { à } 59,62 \text { à } \\
65,68,69,72,73,76 \text { à } 78,81 \text { à } \\
83,85,86,88,90,91,96 \text { à } 105\end{array}$ \\
\hline Évaluation & 10 & \\
\hline
\end{tabular}




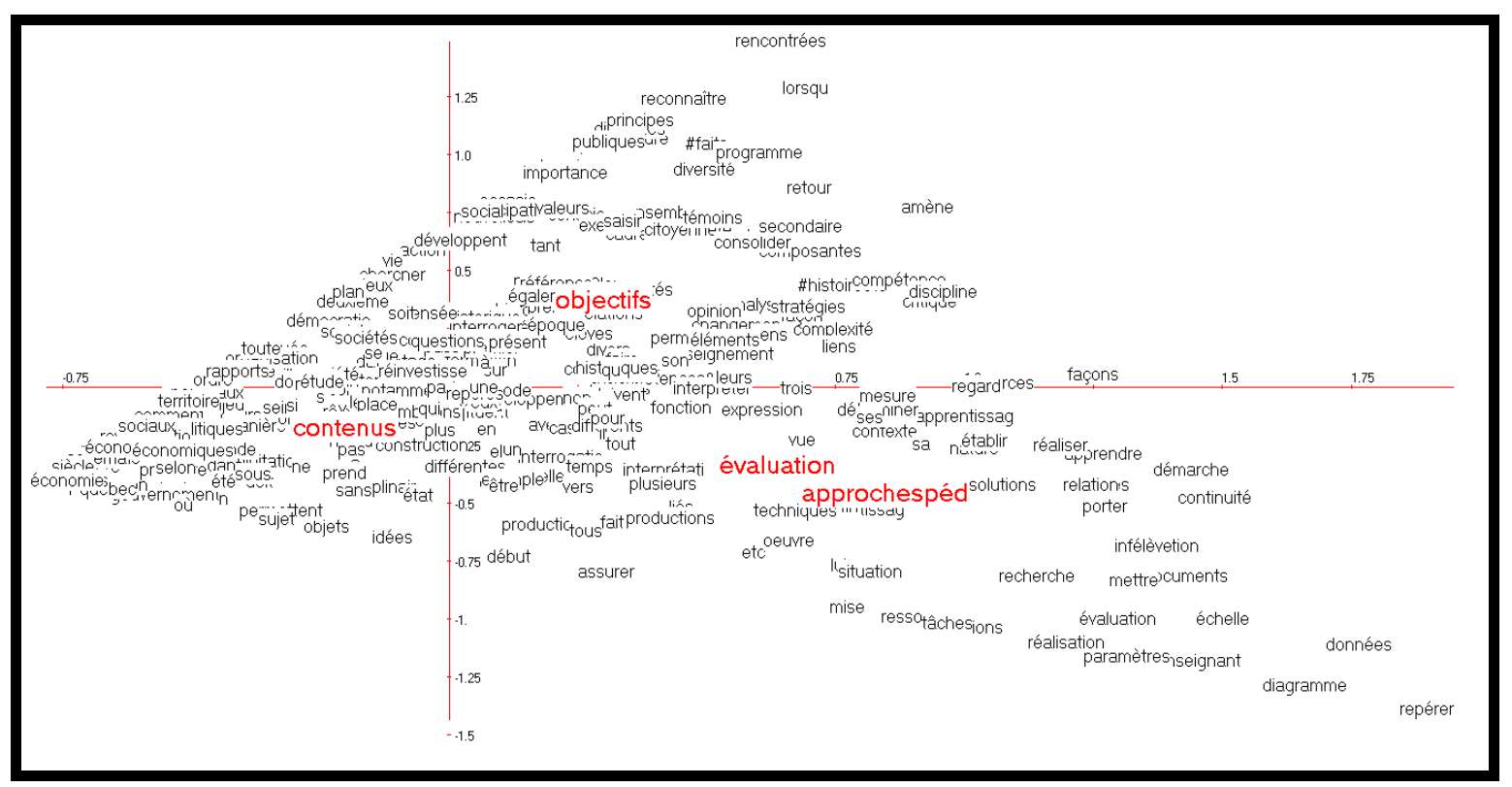

Figure 1. Premier et deuxième axes (corpus Histoire et éducation à la citoyenneté) 
Tableau 2

Contributions à l'axe 1 , représentant $54,33 \%$ de la variance totale

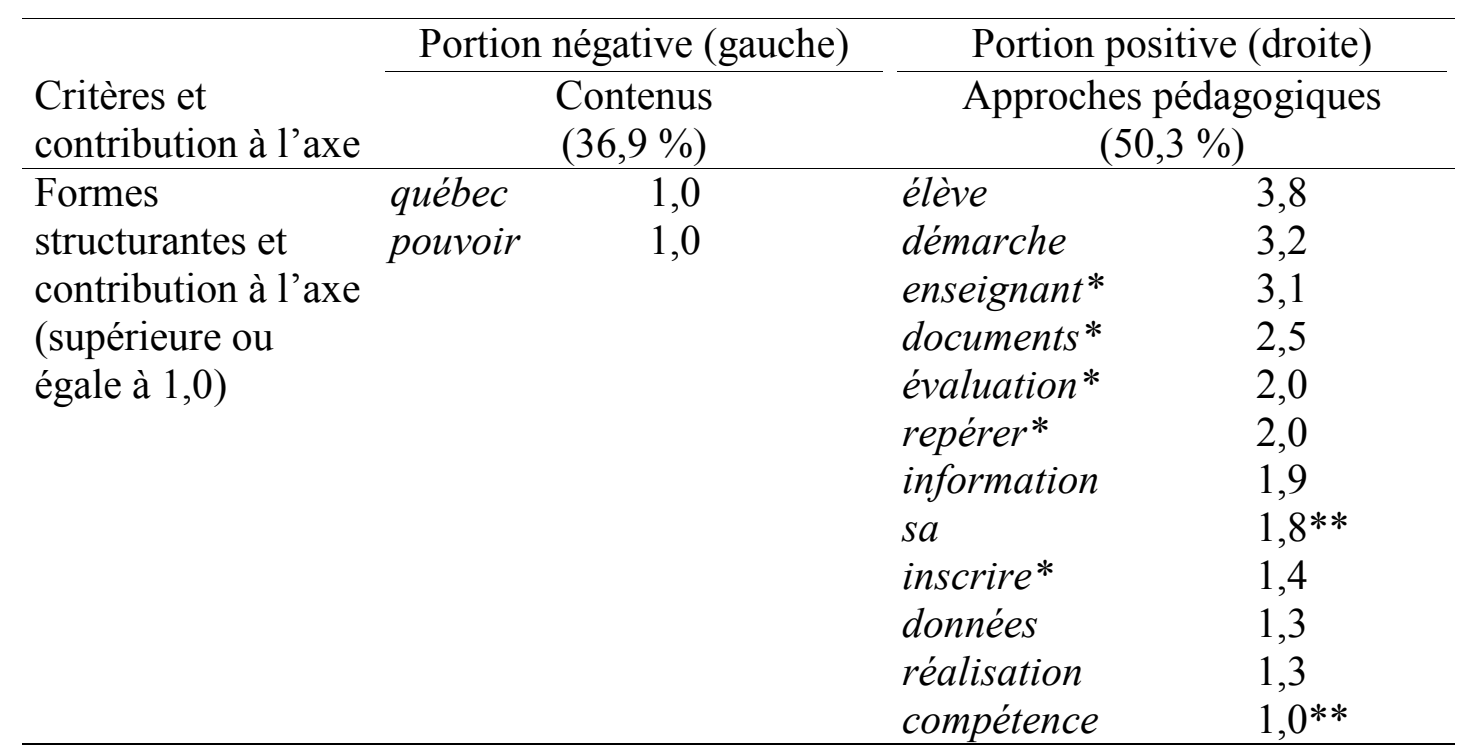

* Formes structurantes qui font l'objet d'un effet de projection dans le plan factoriel. La qualité de leur représentation graphique trahit leur position réelle dans le nuage de points. ** Formes validées au troisième axe (figure 4). 


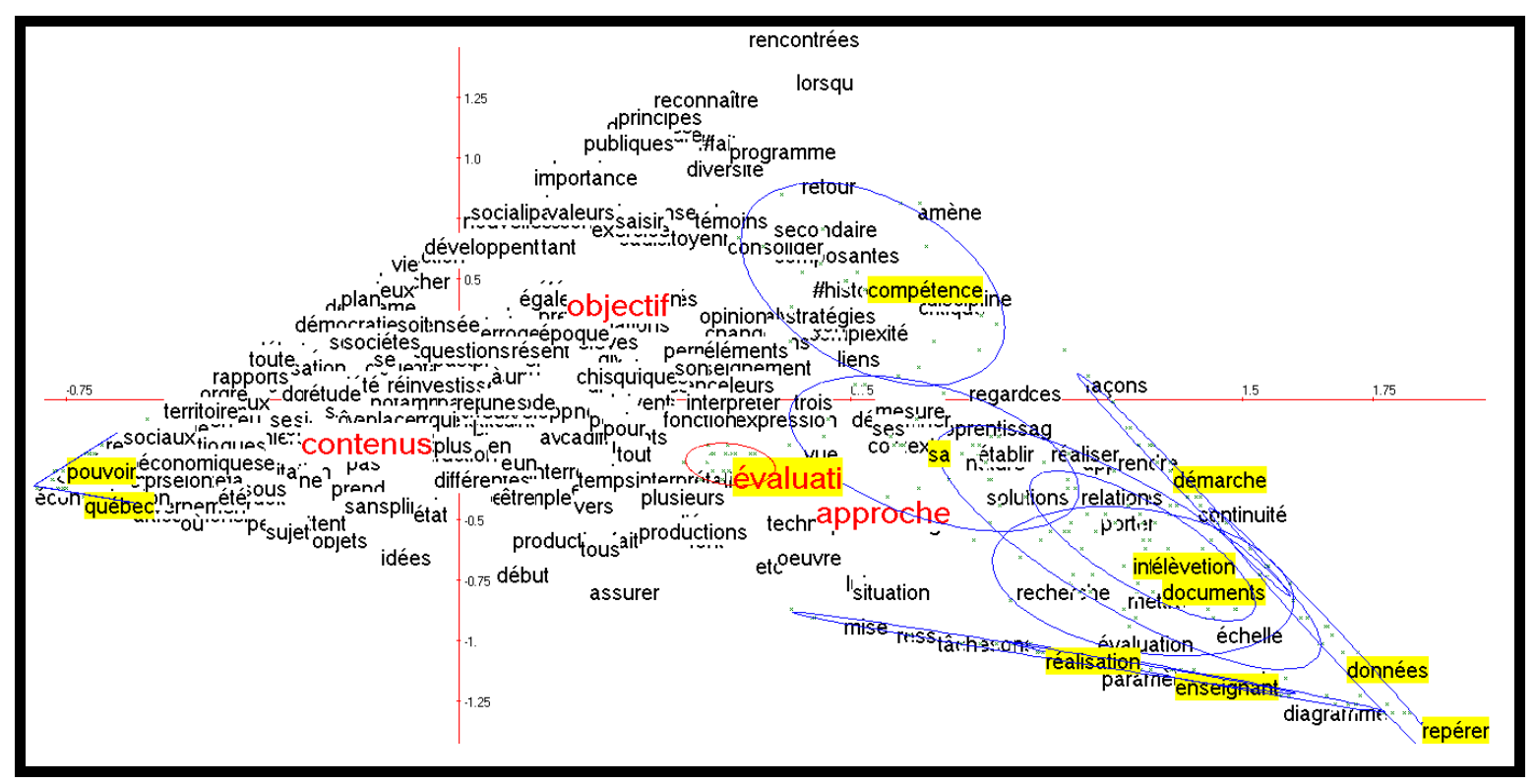

Figure 2. Validation des formes lexicales contribuant à l'inertie du premier axe 
Tableau 3

Contributions à l'axe 2 , représentant $32,88 \%$ de la variance totale

\begin{tabular}{|c|c|c|c|c|}
\hline \multirow[b]{2}{*}{$\begin{array}{l}\text { Critères et } \\
\text { contribution à l'axe }\end{array}$} & \multicolumn{2}{|c|}{ Portion négative (inférieure) } & \multicolumn{2}{|c|}{ Portion positive (supérieure) } \\
\hline & \multicolumn{2}{|c|}{$\begin{array}{l}\text { Approches pédagogiques } \\
(26,5 \%)\end{array}$} & \multicolumn{2}{|c|}{$\begin{array}{l}\text { Objectifs } \\
(64,5 \%)\end{array}$} \\
\hline Formes & enseignant* & $3,5 * *$ & programme & 3,3 \\
\hline structurantes et & évaluation* & $2,0 * *$ & leur & 2,5 \\
\hline contribution à l'axe & réalisation & $1,8 * *$ & $i l s$ & 2,1 \\
\hline (supérieure ou & repérer* & $1,8 * *$ & rencontrées* & 1,8 \\
\hline égale à 1,2) & élève & $1,4 * *$ & citoyenneté & 1,6 \\
\hline & documents* & $1,3^{* *}$ & réalités & 1,6 \\
\hline & inscrire* $^{*}$ & $1,2 * *$ & lorsqu & 1,5 \\
\hline & tâches & 1,2 & reconnaître & 1,5 \\
\hline & situations & 1,2 & comprendre & 1,4 \\
\hline & & & sociales & 1,4 \\
\hline & & & exercice & 1,3 \\
\hline & & & difficultés & 1,2 \\
\hline
\end{tabular}

* Formes structurantes qui font l'objet d'un effet de projection dans le plan factoriel. La qualité de leur représentation graphique trahit leur position réelle dans le nuage de points. ** Formes validées au premier axe (figure 2). 


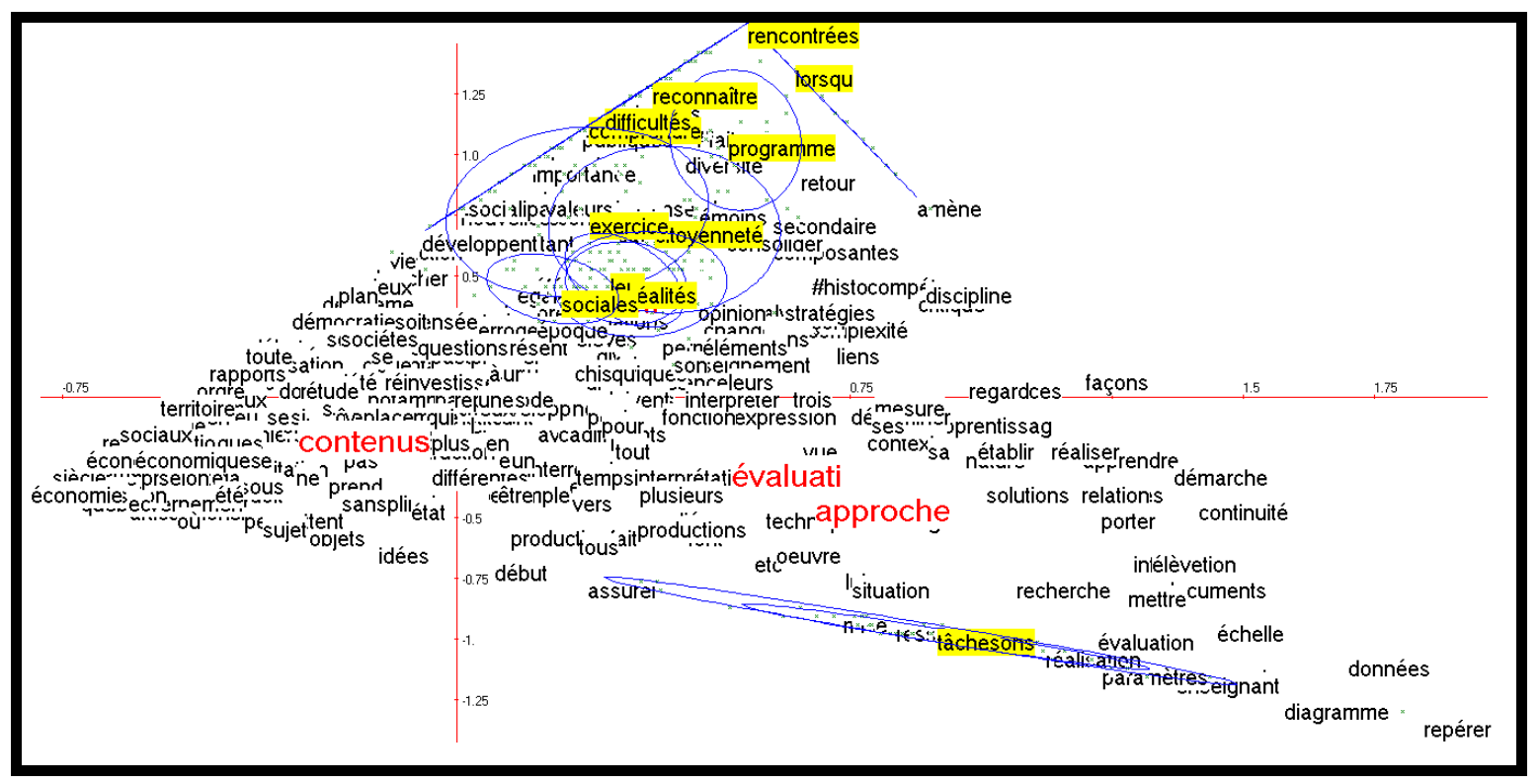

Figure 3. Validation des formes lexicales contribuant à l'inertie du deuxième axe 
Tableau 4

Contributions à l'axe 3 , représentant $12,79 \%$ de la variance totale

\begin{tabular}{|c|c|c|c|c|}
\hline \multirow[b]{2}{*}{$\begin{array}{l}\text { Critères et } \\
\text { contribution à l'axe }\end{array}$} & \multicolumn{2}{|c|}{ Portion négative (inférieure) } & \multicolumn{2}{|c|}{ Portion positive (supérieure) } \\
\hline & \multicolumn{2}{|c|}{$\begin{array}{c}\text { Évaluation } \\
(91,5 \%)\end{array}$} & \multicolumn{2}{|c|}{$\begin{array}{c}\text { Approches pédagogiques } \\
(8,4 \%)\end{array}$} \\
\hline Formes & évaluation* & $18,0 * *$ & documents & $1,8 * *$ \\
\hline structurantes et & composantes* & 6,6 & repérer* & $1,4 * *$ \\
\hline contribution à l'axe & compétences & 4,3 & $s a$ & 1,4 \\
\hline (supérieure ou & analyse & 3,7 & inscrire $^{*}$ & $1,1 * *$ \\
\hline égale à 1,1) & année & 2,0 & & \\
\hline & critique & 2,0 & & \\
\hline & fin & 1,9 & & \\
\hline & situation & 1,8 & & \\
\hline & cadre & 1,6 & & \\
\hline & compte & 1,6 & & \\
\hline & apprentissage & 1,4 & & \\
\hline & permet & 1,3 & & \\
\hline & compétence & 1,1 & & \\
\hline & historiques & 1,1 & & \\
\hline
\end{tabular}

* Formes structurantes qui font l'objet d'un effet de projection dans le plan factoriel. La qualité de leur représentation graphique trahit leur position réelle dans le nuage de points. ** Formes validées au premier axe (figure 2). 


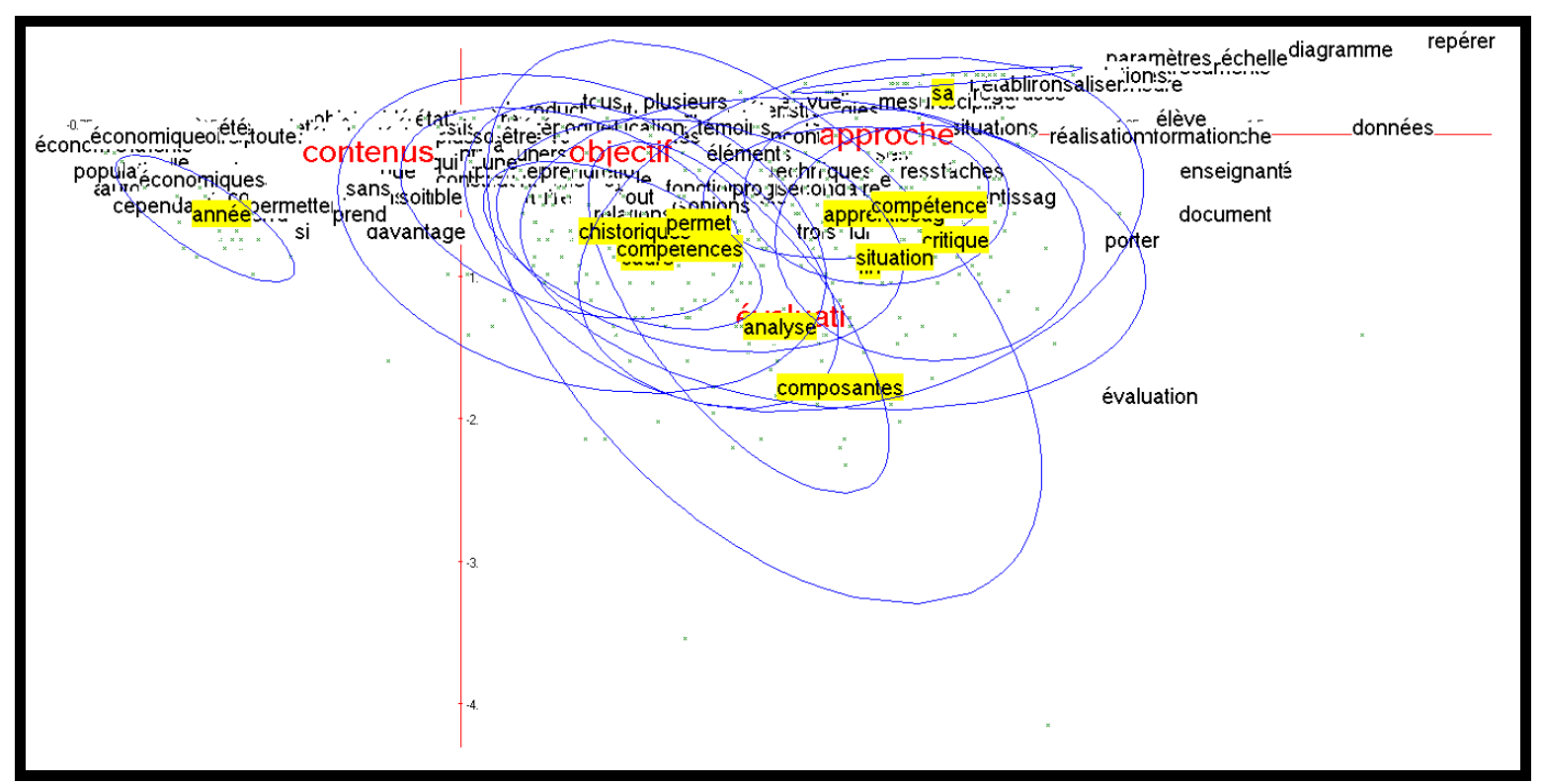

Figure 4. Validation des formes lexicales contribuant à l'inertie du troisième axe 